\title{
CORRECTION
}

View Article Online

View Journal I View Issue

\section{Correction: Design of a robust superhydrophobic surface: thermodynamic and kinetic analysis}

Cite this: Soft Matter, 2015,

11, 3733

Anjishnu Sarkar* and Anne-Marie Kietzig

Correction for 'Design of a robust superhydrophobic surface: thermodynamic and kinetic analysis'

DOI: $10.1039 / c 5 s m 90064 f$

by Anjishnu Sarkar et al., Soft Matter, 2015, 11, 1998-2007.

www.rsc.org/softmatter

The authors would like to correct some errors in eqn (27), (28) and (30)-(32).

Eqn (27) should read as follows:

$$
h_{\mathrm{WSDD}, \text { static }}=\frac{2 \gamma_{L A}}{\rho g R}+\frac{4 \gamma_{L A} a \cos \theta_{Y}}{\rho g b(2 a+b)}
$$

Eqn (28) should read as follows:

$$
h_{\mathrm{WSDD}, \text { dynamic }}=7.53 \times 10^{-4} \frac{c_{1} v}{g}+0.5 \frac{v^{2}}{g}+\frac{4 \gamma_{L A} a \cos \theta_{Y}}{\rho g b(2 a+b)}\left(1-\frac{2.57 \times 10^{-7}}{N m^{-2}} . \rho c_{1} v\right)
$$

Eqn (30)-(32) should read as follows:

The quadratic term of the velocity corresponding to the Bernoulli pressure has been ignored.

$$
v_{\text {calc }}=\frac{1}{\rho c_{1}} \frac{P_{\text {antiwetting }}}{\left(7.53 \times 10^{-4}+P_{\text {antiwetting }}\left(\frac{2.57 \times 10^{-7}}{N m^{-2}}\right)\right)}
$$

Square micropillars:

$$
v_{\text {calc }}=\frac{1}{\rho c_{1}} \frac{4 \gamma_{L A}\left|\cos \theta_{Y}\right|}{a\left(\left(1+\left(\frac{b}{a}\right)_{\exp }\right)^{2}-1\right)\left(7.53 \times 10^{-4}+\frac{4 \gamma_{L A}\left|\cos \theta_{Y}\right|}{a\left(\left(1+\left(\frac{b}{a}\right)_{\exp }\right)^{2}-1\right)}\left(\frac{2.57 \times 10^{-7}}{N m^{-2}}\right)\right)}
$$

Cylindrical micropillars:

$$
v_{\mathrm{calc}}=\frac{1}{\rho c_{1}} \frac{\pi \gamma_{L A}\left|\cos \theta_{Y}\right|}{a\left(\left(1+\left(\frac{b}{a}\right)_{\exp }\right)^{2}-1\right)\left(7.53 \times 10^{-4}+\frac{\pi \gamma_{L A}\left|\cos \theta_{Y}\right|}{a\left(\left(1+\left(\frac{b}{a}\right)_{\exp }\right)^{2}-1\right)}\left(\frac{2.57 \times 10^{-7}}{N m^{-2}}\right)\right)}
$$

The Royal Society of Chemistry apologises for these errors and any consequent inconvenience to authors and readers. 